\title{
BIOFILM PRODUCTION BY CLINICAL STAPHYLOCOCCI STRAINS FROM CANINE OTITIS
}

\section{Camila Alencar Moreira ${ }^{1 *}$, Lis Christina de Oliveira ${ }^{1}$, Marina Silveira Mendes ${ }^{1}$, Thiago de Melo Santiago ${ }^{2}$, Eduardo Bedê Barros $^{2}$, Cibele Barreto Mano de Carvalho ${ }^{1}$}

${ }^{1}$ Laboratório de Bacteriologia, Centro de Biomedicina, Departamento de Patologia e Medicina Legal, Universidade Federal do Ceará, Fortaleza, CE, Brasil; ${ }^{2}$ Laboratório de Microscopia Avançada, Departamento de Física, Universidade Federal do Ceará, Fortaleza, CE, Brasil.

Submitted: November 23, 2010; Returned to authors for corrections: February 08, 2011; Approved: August 15, 2011.

\begin{abstract}
This study determined the species of 54 staphylococci isolates from canine otitis and their ability to produce biofilm through the Congo red agar method, confirmed by scanning electron microscopy. The most frequently identified species were S. intermedius and S. simulans. Results showed that $30 \%$ of the strains were biofilm producers.
\end{abstract}

Key words: biofilm, Congo red agar, scanning electronic microscopy, canine otitis

Since the initial observations that some staphylococcal strains produced biofilm, a great deal of research has been carried out to evaluate its clinical significance. Hitherto there are many reports of biofilm production amidst clinically relevant staphylococci $(1,2)$, often citing this characteristic as an important virulence factor (4). Bacteria from the genus Staphylococcus, including Staphylococcus intermedius and Staphylococcus simulans, are reported to constitute normal skin flora, but also to be involved in severe infections in both humans and animals (1, 5, 7, 11). Among the many staphylococcal infections in veterinary medicine, otitis is the most common ear disease in dogs and may present itself with high levels of antimicrobial resistance, when of an infectious nature (7), a characteristic that is also commonly found in infections associated with biofilm formation (9). Thus, the phenotype differentiation between biofilm producers and nonproducers can help elucidate its impact in veterinary infections $(1,4)$.

This study aims to identify down to species level staphylococcal strains isolated from canine clinical specimens and determine the ability of these strains to produce biofilm.

Fifty-four clinical isolates previously identified by Gram, by morphological and by biochemical characteristics as staphylococci strains (6), were successfully obtained from the collection of the Bacteriology Laboratory at the Federal University of Ceará, Brazil. These isolates were originally acquired from dogs diagnosed with infectious otitis, according to Harvey et al. (3). Species identification was determined through the automated system Vitek2® (bioMérieux, Marcy l'Étoile, France - software 4.03). The two most frequently

\footnotetext{
*Corresponding Author. Mailing address: Laboratório de Bacteriologia, Centro de Biomedicina, Departamento de Patologia e Medicina Legal, Universidade Federal do Ceará, Rua Monsenhor Furtado s/n, Rodolfo Teófilo, CEP 60441-750, Fortaleza - CE, Brasil.; Tel.: +55 85 33668638 Fax: +55 85 $33668300 . ;$ E-mail: milalencar@gmail.com
} 
identified staphylococcus species were $S$. intermedius $(\mathrm{n}=28)$, a species commonly found in animals, especially dogs, usually causing infection (5), and S. simulans $(\mathrm{n}=22)$, a species occasionally found on animal skin and a known opportunistic pathogen (11) (Table 1).

Table 1. Distribution of identified staphylococcal species and phenotypical detection of biofilm-producing strains.

\begin{tabular}{lccc}
\hline Bacterial Isolates & \multicolumn{2}{c}{ CRA* } & \multirow{2}{*}{ Total (\%) } \\
\cline { 2 - 3 } & BP† (\%) & NBP\$ (\%) & $28(52)$ \\
Staphylococcus intermedius & 11 & 17 & $22(40)$ \\
Staphylococcus simulans & 5 & 17 & $2(4)$ \\
Staphylococcus haemolyticus & 0 & 2 & $1(2)$ \\
Staphylococcus epidermidis & 0 & 1 & $1(2)$ \\
Staphylococcus lugdunensis & 0 & 1 & $54(100)$ \\
Total & $16(30)$ & $38(70)$ & \\
\hline
\end{tabular}

*CRA - Congo red agar † BP - Biofilm producer ₹ NBP - Non-biofilm producer

Two strains were used as reference for biofilm production: Staphylococcus aureus ATCC 29523 as positive control and Staphylococcus epidermidis ATCC 12228 as negative control $(1,9)$.

All 56 strains, 54 clinical isolates and the two controls, were submitted to phenotypic characterization of biofilm production using the Congo red agar (CRA) method (2). CRA plates were prepared by adding $0.8 \mathrm{~g}$ of Congo red dye and $36 \mathrm{~g}$ of saccharose (both from Sigma, Missouri, USA) to $1 \mathrm{~L}$ of brain-heart infusion (BHI) agar (Oxoid, Cambridge, UK). Plates with the medium were inoculated and incubated in aerobic conditions for 24 to 48 hours at $37^{\circ} \mathrm{C}$. Black to almost black colored colonies were considered as biofilm producer strains, while red to bordeaux colored colonies on CRA were classified as strains unable to produce biofilm $(1,2)$. The two reference strains S. epidermidis ATCC 12228 and S. aureus ATCC 29523 were found to be, respectively, negative (Figure 1A) and positive (Figure 1D) to the test, as expected.

Among the 54 clinical strains, the final classification was as follows: 16 with black to almost black colonies as biofilm producers and 38 with red to bordeaux colonies as non producers (Table 1). To confirm these findings, the studied strains were cultured and had their images captured by scanning electron microscopy (SEM). Briefly, the staphylococci were incubated for $48 \mathrm{~h}$ at $37^{\circ} \mathrm{C}$ in $\mathrm{BHI}$ broth, without agitation, in 24-well plates and in the presence of a sterile glass slide (diameter $5 \mathrm{~mm}$ ) for each well. The growth medium was discarded and replaced after 24 hours (9). After incubation, each glass slide, with any adherent bacteria, was washed three times for $15 \mathrm{~min}$ each in $0.05 \mathrm{M}$ PBS. Then they were all fixed with $2 \%(\mathrm{wt} / \mathrm{vol})$ glutaraldehyde in filtersterilized 0.05M PBS ( $\mathrm{pH} 7.4)$, at room temperature, for 16 hours. Thereafter, they were rinsed again in sterile PBS solution, in the same manner as before. Following these procedures, the slides were dehydrated in successive ethanolwater mixtures with increasing ethanol concentrations $(20 \%$, $40 \%, 60 \%, 80 \%$, and $95 \%$ [vol/vol] ) for $15 \mathrm{~min}$ each and then twice in absolute ethanol for the same time (10). After drying, samples were affixed to aluminum mounts with double sided carbon adhesive tape and sputter coated with gold. The upper surfaces of the slides were examined by SEM for the presence of biofilm structures and photographed (10) (Figure 1E-H). The negative control and non-biofilm producer strains failed to colonize the surface of the glass slide. Artifacts from the fixation process and small clusters of adhered cells were observed, but these cells did not aggregate to form a monolayer or any form of biofilm structure (Fig. 1E and 1F). The positive control strain and biofilm producer strains grew evident biofilm 
biomass with a multilayered three-dimensional structure (Fig. $1 \mathrm{G}$ and $1 \mathrm{H})$.

Several studies, as the current one, have shown a strong association between $S$. intermedius and canine otitis $(7,8)$. It was the most prevalent species amongst the isolated bacteria, accounting for $52 \%$ of the identified strains in this study. In small animal practice, common infections, such as otitis, often receive empirical antimicrobial therapy before test results are available. This information corroborates that, for the veterinarian, prior knowledge of which bacterial species are most frequently found in each infectious disease is extremely beneficial (8).

The phenotypic detection of biofilm production through cultures on CRA medium (Table 1) was determined after a final evaluation at the end of a $48 \mathrm{~h}$ incubation period (Figure 1A-D). Consistent with findings of Arciola et al. (1), the first reading after $24 \mathrm{~h}$ of incubation in CRA medium was enough to permit a definite result for most strains. In these experiments, only three strains required a second reading at 48 $\mathrm{h}$ to achieve a precise result. However, for all three, the lapse of time between the two readings served only to intensify the colors of the colonies, making the results more pronounced but not changing them. These three were S. simulans strains and were ultimately classified as biofilm producers. Overall, $30 \%$ of the 54 clinical isolates turned out to be biofilm producer strains. The images acquired by SEM supported the CRA test results.

Bacterial infection and biofilms remain as major challenges in modern clinical microbiology (1), and to our knowledge, only a few in vitro studies have addressed the subject of biofilm production in the field of veterinary medicine. This is the first time that $S$. simulans is shown to produce biofilm as an isolated species, and not in a multispecies model. The confirmation of the presence of such virulence factor amongst these staphylococcal strains brings light to the possible role that biofilm formation has in canine otitis. This should be further investigated, possibly to determine its influence on antimicrobial resistance.

In conclusion, we believe that the accurate identification of biofilm producers in clinical specimens may provide greater understanding and help improve the management of staphylococci infections in veterinary medicine.

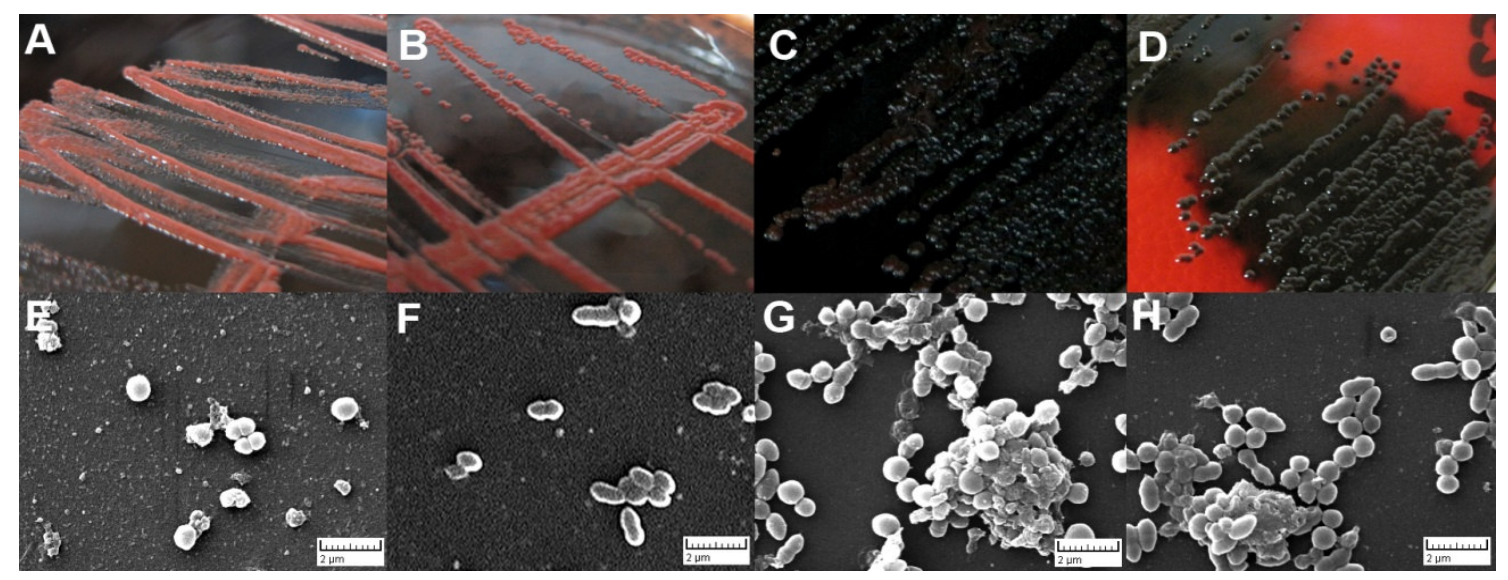

Figure 1. Corroborative Congo red agar (CRA) plate test and scanning electron microscopy (SEM) results for four strains. CRA plate test images for the negative control (A), a non-biofilm producer strain (B), a biofilm producer strain (C) and the positive control (D). Positive strains give black colonies and negative strains give red colonies on CRA. SEM images of Staphylococci strains growing on glass slides after 48 hours for the negative control $(\mathrm{E})$, a non-biofilm producer strain $(\mathrm{F})$, a biofilm producer strain $(\mathrm{G})$ and the positive control $(\mathrm{H})$. Adhered bacteria were fixed, treated and observed by SEM (x10000 magnification). Positive strains show larger cell clusters and biofilm matrix, while the negative strains show only a few adhered cells. Amorphous structures in Fig. 1F are artifacts of fixation as seen in blank slides that went through the same process. Images represent typical fields of view. 


\section{ACKNOWLEDGEMENTS}

This study was supported by CAPES.

\section{REFERENCES}

1. Arciola, C.R., Campoccia, D., Gamberini, S., Cervellati, M., Donati, E., Montanaro, L. (2002). Detection of slime production by means of an optimised Congo red agar plate test based on a colourimetric scale in Staphylococcus epidermidis clinical isolates genotyped for ica locus. Biomaterials. 23, 4233-4239.

2. Freeman, D.J.; Falkiner, F.R.; Keane, C.T. (1989). New method for detecting slime production by coagulase negative staphylococci. J. Clin. Pathol. 42, 872-874.

3. Harvey, R.G.; Harari, J.; Delauche, A. J. (2004). Doenças do ouvido em cães e gatos. Editora Revinter, Rio de Janeiro.

4. Jain, A.; Agarwal, A. (2009). Biofilm production, a marker of pathogenic potential of colonizing and commensal staphylococci. J. Microbiol Meth. $76,88-92$

5. Kempker, R.; Mangalat, D.; Kongphet-Tran, T.; Eaton, M. (2009). Beware of the pet dog: a case of Staphylococcus intermedius infection. Am. J. Med. Sci. 338, 425-427.
6. Murray, P.R.; Baron, E.J.; Jorgensen, J.H.; Pfaller, M.; Yolken, R. (2003). Manual of Clinical Microbiology, 8th Edition. ASM Press, Washington, DC.

7. Oliveira, L.C.; Leite, C.A.L.; Brilhante, R.S.N.; Carvalho, C.B.M. (2008). Comparative study of the microbial profile from bilateral canine otitis externa. Can. Vet. J. 49, 785-788.

8. Pedersen, K.; Pedersen, K.; Jensen, H.; Finster, K.; Jensen, V.F.; Heuer, O.E. (2007). Occurrence of antimicrobial resistance in bacteria from diagnostic samples from dogs. J Antimicrob. Chemother. 60, 775-781.

9. Pettit, R.K.; Weber, C.A.; Kean, M.J.; Hoffmann, H.; Pettit, J.R.; Tan, R.; Franks, K.S.; Horton, M.L. (2005). Microplate Alamar Blue Assay for Staphylococcus epidermidis Biofilm Susceptibility Testing. Antimicrob. Agents. Ch. 49, 2612-2617.

10. Tran, P.L.; Hammond, A.A.; Mosley, T.; Cortez, J.; Gray, T.; ColmerHamood, J.A.; Shashtri, M.; Spallholz, J.E.; Hamood, A.N.; Reid, T.W. (2009). Organoselenium coating on cellulose inhibits the formation of biofilms by Pseudomonas aeruginosa and Staphylococcus aureus. Appl. Environ. Microb. 75, 3586-3592.

11. Vallianou, N.; Evangelopoulos, A.; Makri, P.; Zacharias, G.; Stefanitsi, P.; Karachalios, A.; Avgerinos, P.C. (2008). Vertebral osteomyelitis and native valve endocarditis due to Staphylococcus simulans: a case report. J. Med. Case Rep. http://www.jmedicalcasereports.com/content/2/1/183 (doi:10.1186/1752-1947-2-183) 